\title{
Embarazo ectópico cervical: diagnóstico preciso y enfoque de manejo médico. Reporte de caso
}

\section{Ectopic Cervical Pregnancy - Precise Diagnosis and Medical Management Approach. Case Report}

\author{
Rodolfo A. Martínez D. a \\ Pontificia Universidad Javeriana, Colombia \\ Leila Quintero \\ Pontificia Universidad Javeriana, Colombia \\ Carlos A. García \\ Pontificia Universidad Javeriana, Colombia \\ Alfredo Fernández de Castro \\ Pontificia Universidad Javeriana, Colombia
}

a Autor de correspondencia. Correo electrónico: rodolfo.martinez@javeriana.edu.co

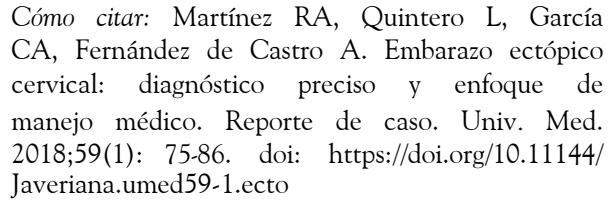

Cómo citar: Martínez RA, Quintero L, García CA, Fernández de Castro A. Embarazo ectópico cervical: diagnóstico preciso y enfoque de manejo médico. Reporte de caso. Univ. Med. 2018;59(1): 75-86. doi: https://doi.org/10.11144/ Javeriana.umed59-1.ecto

\section{RESUMEN}

El embarazo ectópico cervical es una condición exótica. Su diagnóstico y manejo plantean un desafío importante, dada su asociación con desenlaces maternos graves. La sospecha clínica y la juiciosa aplicación de los criterios diagnósticos permiten establecer modalidades de terapia conservativa. Este artículo reporta un caso de embarazo ectópico cervical con diagnóstico de imágenes de ultrasonido y resonancia nuclear magnética en el que se administró tratamiento con metotrexato. El seguimiento clínico, de los exámenes paraclínicos y de ultrasonido mostraron la resolución completa de la condición. Se revisan aspectos relacionados con el diagnóstico y tratamiento.

Palabras clave

embarazo ectópico; cuello del útero; ultrasonografía; espectroscopia de resonancia magnética; metotrexato.

\section{ABSTRACT}

Cervical ectopic pregnancy is an exotic condition. Its diagnosis and management pose a major challenge given its association with serious maternal outcomes. The clinical suspicion and the judicious application of the diagnostic criteria allow for conservative therapy modalities. In this article, a case of cervical ectopic pregnancy is reported to diagnostic ultrasound imaging and nuclear magnetic resonance in which treatment with Methotrexate was administered. Clinical follow-up of the laboratory test and ultrasound showed complete resolution of the condition. Aspects related to the diagnosis and treatment are reviewed.

Keywords

pregnancy ectopic; ultrasonography; magnetic resonance spectroscopy; Methotrexate. 


\section{Introducción}

El embarazo ectópico (EE) se define como:

[...] una condición potencialmente mortal en la que la implantación del embrión se produce fuera de la cavidad del útero. La mayoría de los EEs se producen en las trompas de Falopio y son conocidos como Embarazo Ectópico Tubárico. Sin embargo la implantación se puede dar en otros sitios anatómicos, como el cuello uterino (embarazo ectópico cervical), los ovarios (embarazo ectópico ovárico) y en la cavidad abdominal (embarazo abdominal). (1)

El EE es una condición clínica grave y potencialmente letal. Su frecuencia es creciente: se ha reportado un estimado de 8,6 (1988-2008) a 11,1 (2003-2005) de EE por cada 1000 embarazos en el Reino Unido (2), y en Estados Unidos, el Centro de Control de Enfermedades reportaba para 1992 un número de 108.800 casos, cifra muy superior a los 17.800 estimados para 1970, y que corresponde a una rata de 19,7 EE por cada 1000 embarazos reportados (3).

El EE es la causa más importante de muerte materna durante el primer trimestre de la gestación. En efecto, es el responsable de hasta un 13\% de todas las muertes maternas (4) en Estados Unidos; en tanto que en el Reino Unido (2) es responsable del 7,5\% de las causas directas de muerte materna.

En Colombia, es una condición de importancia en salud pública. Ruiz (5) describe que para el 2006, según cifras nacionales, el EE fue causa del 2,05\% de las muertes maternas $(11 / 536)$, y esta cifra es mayor que la reportada para 1998, año en el que las cifras de mortalidad materna ubican el embarazo terminado en aborto/embarazo ectópico como responsable de $1,5 \%$ de este grupo (11/722) (6).

El porcentaje de EE no tubárico es el $5 \%$ del total de las gestaciones ectópicas y el embarazo ectópico cervical (EEC) es una forma muy poco común, donde hay una implantación del blastocisto en la mucosa cervical por debajo del orificio cervical interno; su prevalencia es menor al $1 \%$, y se presenta en aproximadamente $1 / 1000$ a $1 / 95.000$ embarazos $(7,8)$.

En Colombia existen pocos reportes de EE. Gómez Palacino (9) presentó una interesante serie
95 casos en 1954 y no reportaba alguno de tipo ectópico cervical. Se destaca, así mismo, el publicado por Parra Anaya (10), en Barranquilla, en 1993, como coautor del estudio que planteó los criterios de ultrasonido para el diagnóstico de EEC, criterios que a la fecha siguen siendo aceptados en todas las publicaciones.

Posteriormente, en 2011, Herrera (11) reportó sobre una serie de embarazos heterotópicos en la que uno de sus casos incluía un EEC asociado con un procedimiento de tecnología reproductiva.

A continuación, se describe el caso de una paciente con diagnóstico de EEC, tratado en el Hospital Universitario San Ignacio, Bogotá, Colombia, en quien la aproximación diagnóstica se hace de forma eficiente y quien recibe tratamiento médico con una adecuada evolución. El presente artículo fue aprobado por el Comité de Investigación del Hospital Universitario San Ignacio. La paciente firmó el consentimiento para la utilización de los datos de la historia clínica y las imágenes de ultrasonido y resonancia magnética nuclear (RNM). Ningún dato personal se revela en la presente publicación.

\section{Reporte de caso}

El caso corresponde a una mujer de 37 años de edad, natural y procedente de Bogotá, Colombia. Consultó por un cuadro clínico de sangrado vaginal y dolor en el hipogastrio tipo cólico. En el examen físico de ingreso, la especuloscopia mostró una vagina normal con un cuello uterino cuyo labio anterior era prominente, sin evidencia de sangrado; en el tacto vaginal se encontró una vagina normal, así como un cuello uterino largo posterior cerrado. El útero en anteroversoflexión (AVF) era de forma y tamaño normal. El fondo del saco anterior estaba abombado y doloroso, y los anexos no eran palpables. Tenía antecedente de cesárea por embarazo gemelar.

A la paciente se le realizó una prueba en sangre para cuantificar la fracción beta de la gonadotrofina coriónica humana (Abbott Laboratories. Architect.B-hCG Total), que se reportó en $31.819 \mathrm{mUI} / \mathrm{ml}$. También se le tomó una ecografía endovaginal (Toshiba-Xario XG) que mostró la presencia de útero con medidas de $72 \times$ 
$52 \times 54 \mathrm{~mm}$ con un cuello de $35 \mathrm{~mm}$ con orificio interno cerrado. El endometrio midió $18 \mathrm{~mm}$, sin evidencia de saco gestacional intrauterino y en la región ístmica y cervical, por debajo de las arterias uterinas, se encontró un saco gestacional de $30 \mathrm{~mm}$ con presencia de embrión único de $20,6 \mathrm{~mm}$ sin presencia de actividad cardiaca fetal (figuras 1 a 4 ).

\section{Figura 1}

Presencia de cavidad uterina vacia, con endometrio sin presencia de saco gestacional. Presencia de saco gestacional cervical

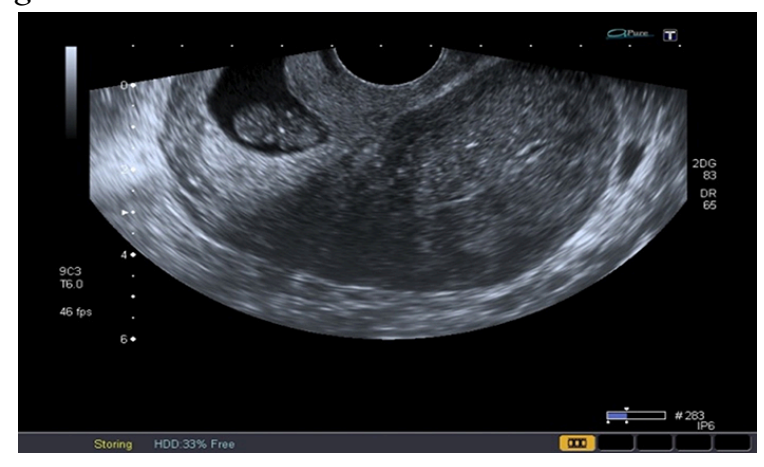

Figura 2

Presencia de cavidad endometrial vacia (flecha amarilla). Embarazo extrauterino cervical con embrión único sin actividad cardiaca (flecha blanca)

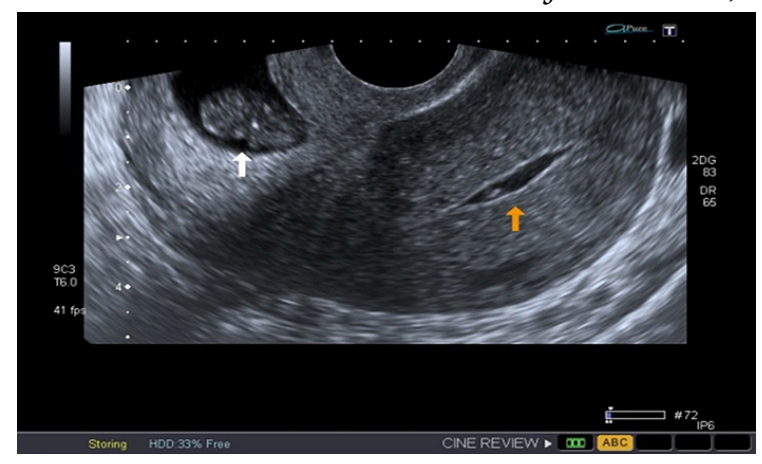

\section{Figura 3}

Polo embrionario de 20,6 $\mathrm{mm}$ sin actividad cardiaca

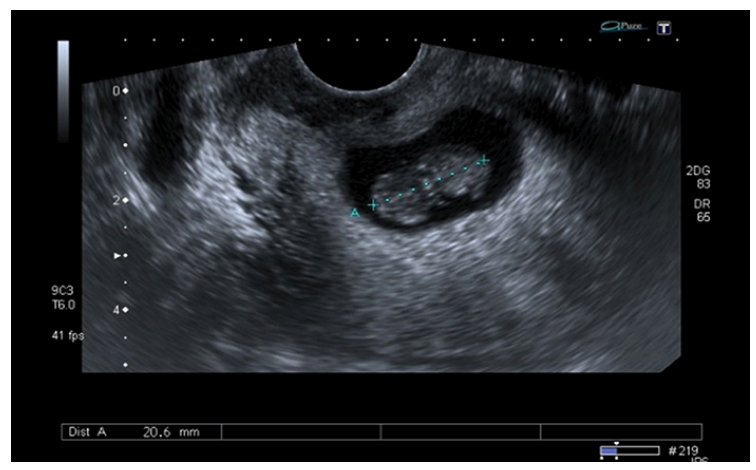

\section{Figura 4}

Orificio cervical interno cerrado (flecha amarilla), debajo del cual se evidencia la implantación del saco gestacional en la mucosa cervical

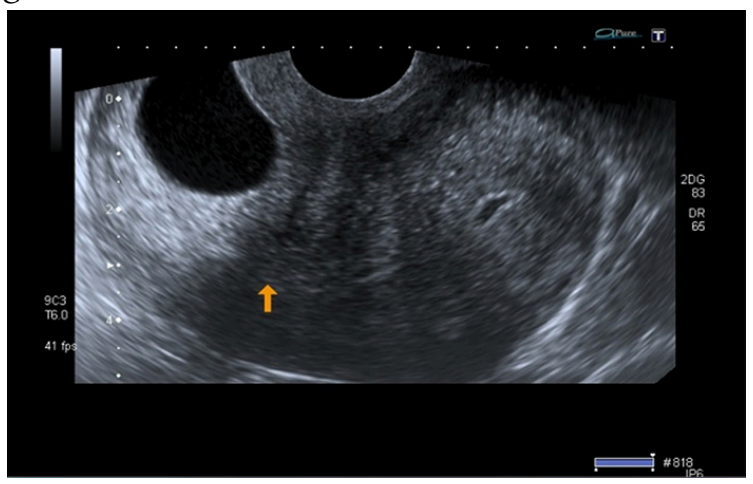

Se realizó una RNM (Philips 1.5 Tesla) que permitió confirmar el diagnóstico de EEC (figuras 5 a 8). 


\section{Figura 5A}

Imágenes sagitales de RMN potenciadas en $T 2$ (TR: 1540 TE: 70) y T2 de disparo único (TR: 709 TE: 315) que muestran el saco gestacional hiperintenso localizado en el canal cervical y con tejido fetoplacentario en su interior, más evidente en la imagen B. Nótese que los orificios cervicales interno $y$ externo se encuentran cerrados

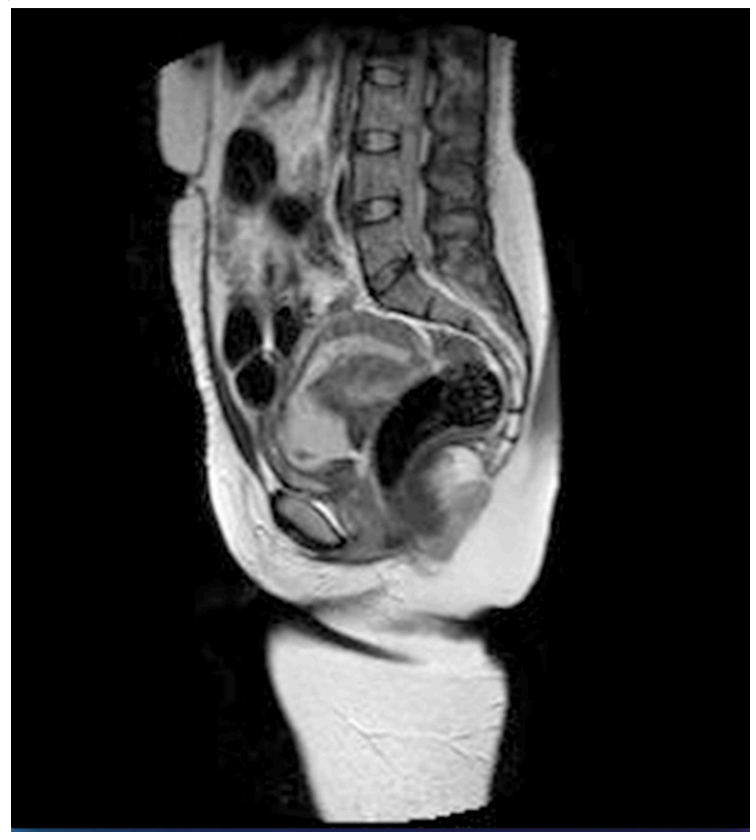

\section{Figura 5B}

Imágenes sagitales de RMN potenciadas en $T 2$ (TR: 1540 TE: 70) y T2 de disparo único (TR: 709 TE: 315) que muestran el saco gestacional biperintenso localizado en el canal cervical y con tejido fetoplacentario en su interior, más evidente en la imagen B. Nótese que los orificios cervicales interno $y$ externo se encuentran cerrados

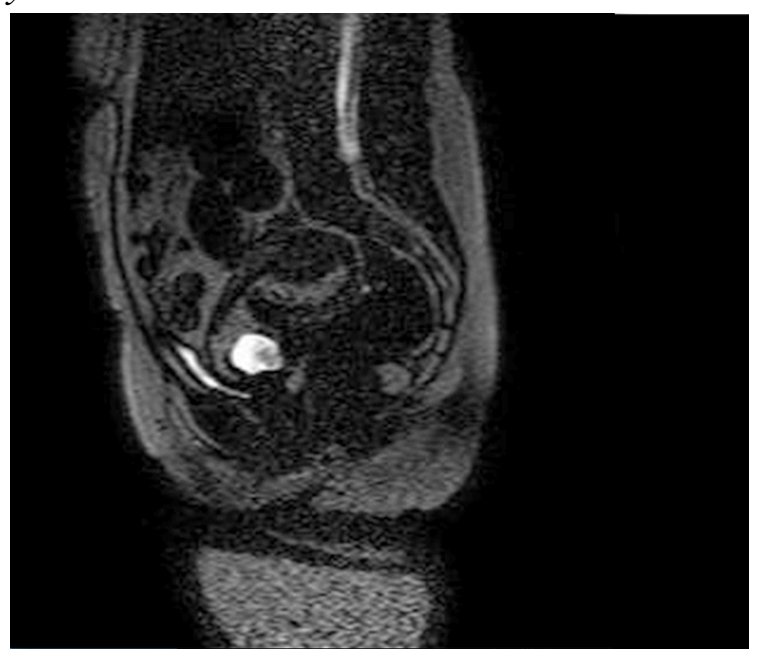

\section{Figura 6}

Imagen sagital de RMN en T2 con supresión grasa (SPIR) que muestra el saco gestacional limitado anteriormente por una banda de señal intermedia correspondiente a la reacción decidual y rodeada a su vez por el miometrio hipointenso

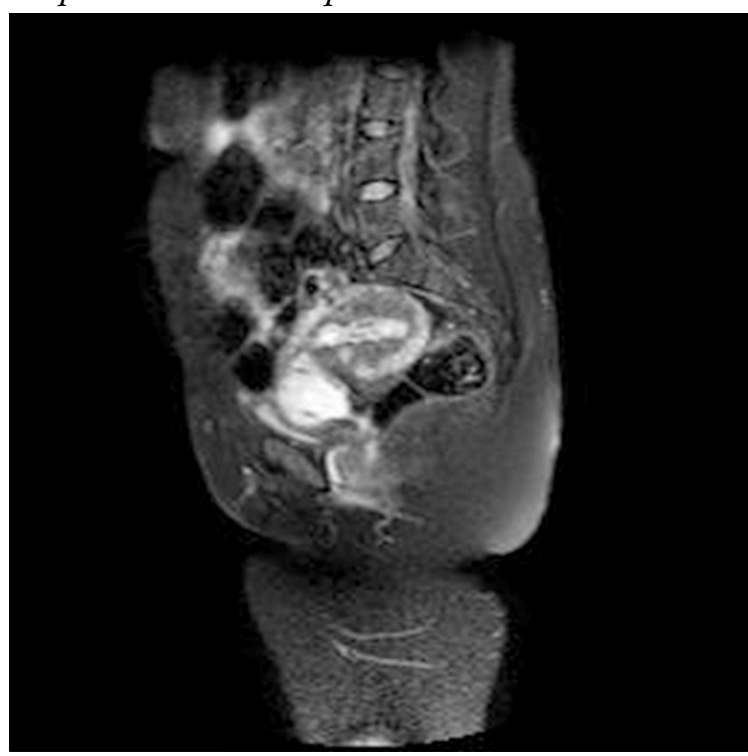




\section{Figura 7A}

Imágenes de $R M N$ potenciadas en $T 2$ con supresión de la grasa. A) Plano axial y B) Plano coronal, donde se identifica el saco gestacional como una estructura quistica con tejido fetoplacentario en su interior y localizado en el canal cervical

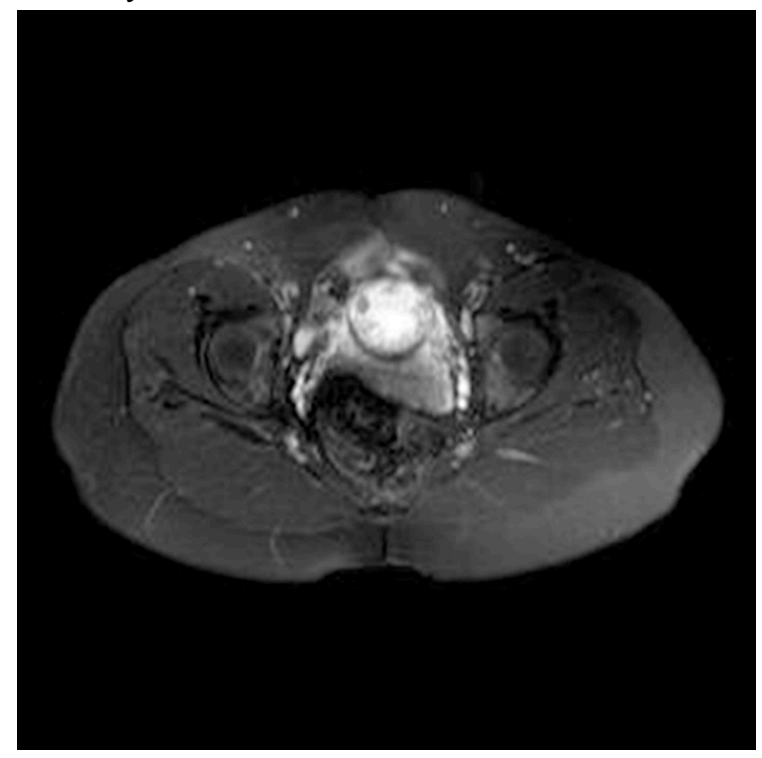

Figura 7B

Imágenes de $R M N$ potenciadas en $T 2$ con supresión de la grasa. A) Plano axial y B) Plano coronal, donde se identifica el saco gestacional como una estructura quistica con tejido fetoplacentario en su interior y localizado en el canal cervical

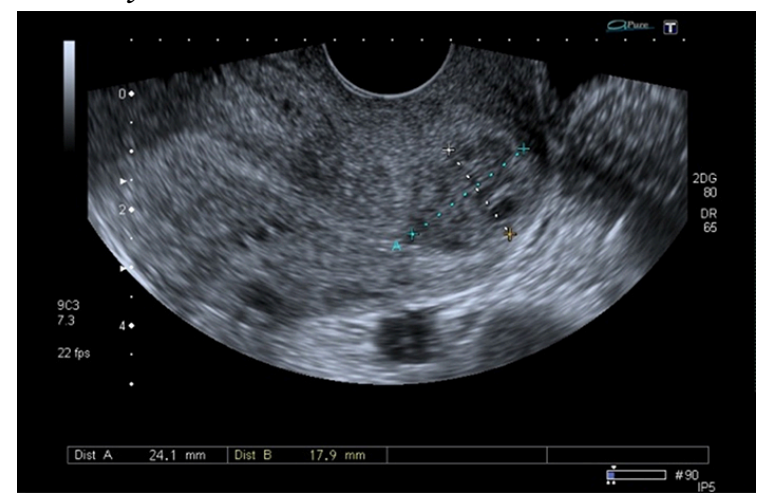

\section{Figura 8A}

A) Imagen sagital de RMN en T1 que muestra el miometrio y el saco gestacional de baja señal, lo cual limita su diferenciación. B). Imagen sagital de T1 con gadolinio y supresión grasa que evidencia el realce normal del miometrio y el saco gestacional hipointenso y sin realce

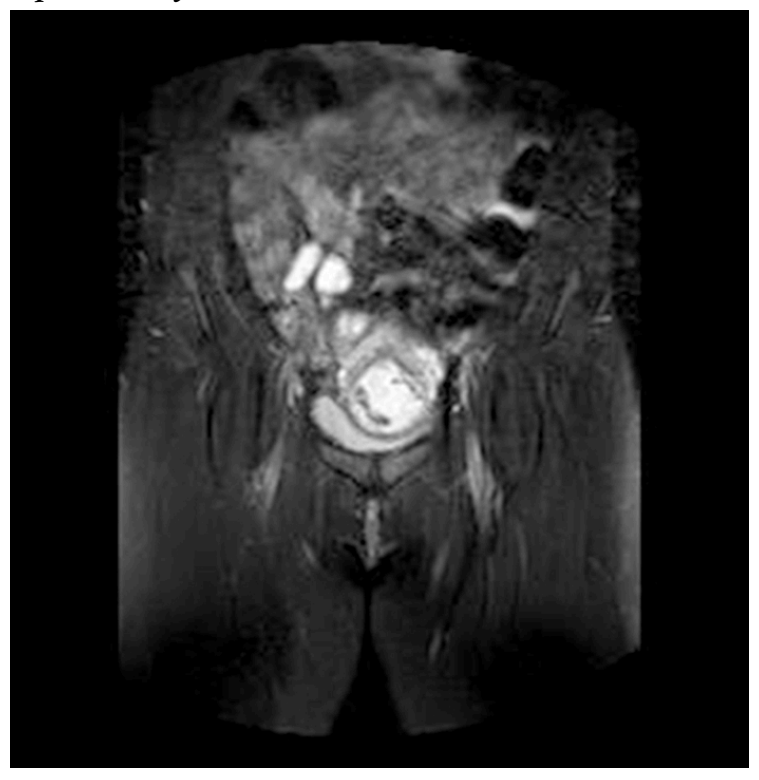




\section{Figura 8B}

A) Imagen sagital de RMN en T1 que muestra el miometrio y el saco gestacional de baja señal, lo cual limita su diferenciación. B). Imagen sagital de T1 con gadolinio y supresión grasa que evidencia el realce normal del miometrio y el saco gestacional bipointenso y sin realce

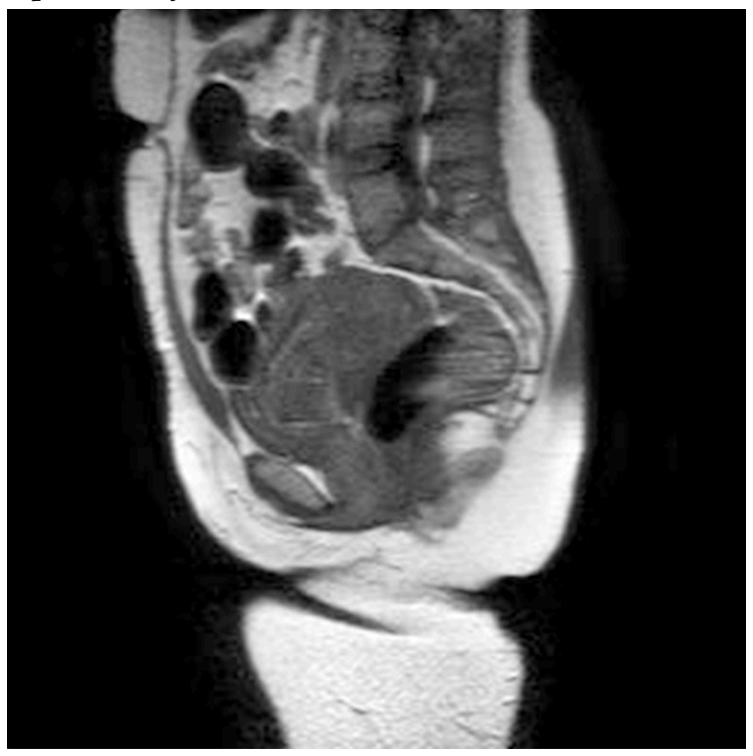

Se trata de una paciente con diagnóstico de EEC, estable hemodinámicamente, sin dolor, con deseos de paridad. Por lo tanto, se decidió iniciar tratamiento conservador con metotrexato en un esquema de dosis múltiple a dosis de $1 \mathrm{mg} / \mathrm{kg} /$ día, los días 1, 3, 5 y 7, con administración ácido folínico los días 2, 4, 6 y 8. Se hospitalizó en la Unidad Materno Fetal de nuestra institución para observación clínica y aplicación del tratamiento instaurado.

La paciente mostró una evolución satisfactoria, con descenso progresivo de las concentraciones beta de la gonadotrofina coriónica humana hasta 23 $\mathrm{mUI} / \mathrm{ml}$, momento en el cual se dio de alta con control ambulatorio para seguimiento ecográfico.

A los dos meses asistió a control y se le realizó una ecografía que mostró la presencia de una imagen hipoecoica heterogénea en la zona del istmo de 2 $\mathrm{cm}$ de diámetro en el labio anterior, sin presencia de embrión o saco gestacional (figuras 9 y 10).
Figura 9

Presencia de imagen hipoecoica en el labio anterior del cuello uterino que corresponde al tejido cicatricial.

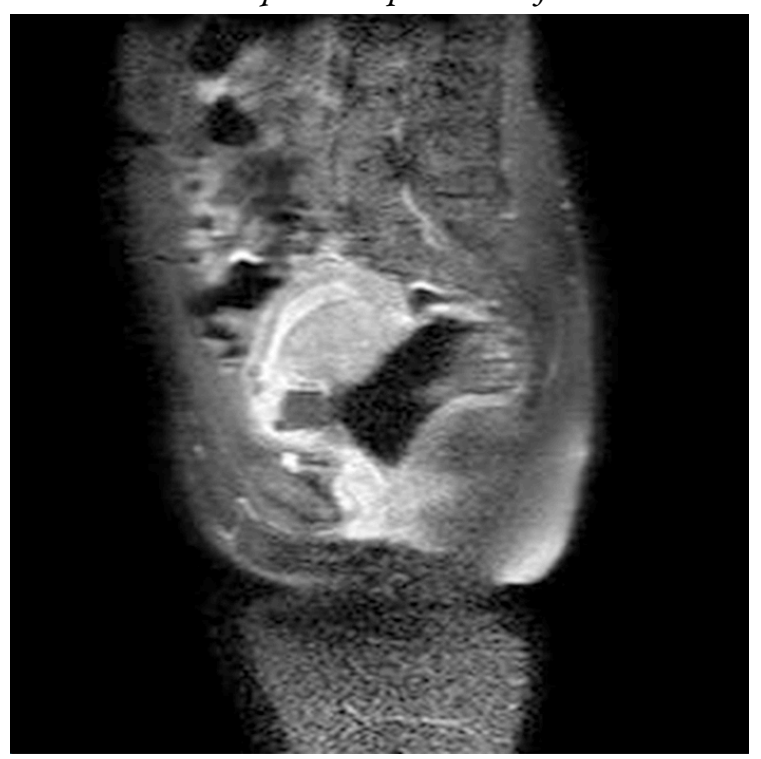

\section{Figura 10}

Imagen hipoecoica en el labio anterior de 24,1 $\times$ $17,9 \mathrm{~mm}$

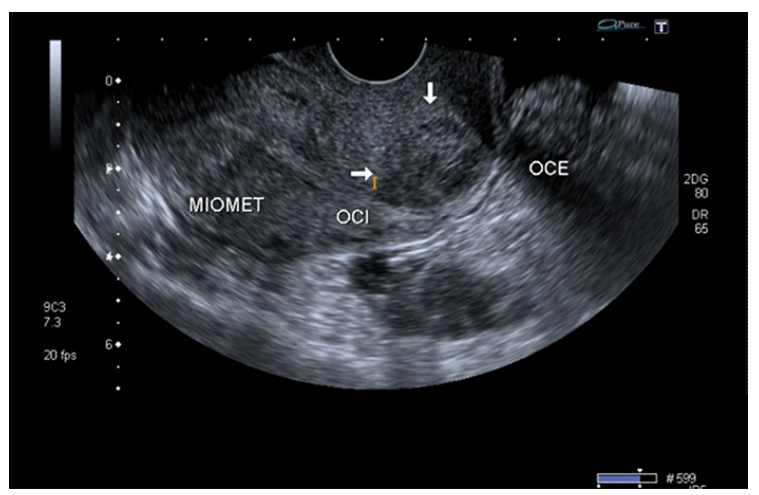

La paciente únicamente presentó un sangrado escaso durante el proceso de manejo inicial y el periodo poshospitalización. Posteriormente fue dada de alta.

\section{Discusión}

ElEEC es una condición clínica muy poco frecuente. La etiología es desconocida, y si bien más del 90\% de los casos se presenta sin antecedentes relevantes, según la serie de Verma y cols. (12) existen algunos factores de riesgo descritos en la 
literatura afines a procedimientos de tecnología reproductiva como son la transferencia intratubárica de gametos (13), la inseminación intrauterina (14), al igual que procedimientos quirúrgicos como ablación endometrial (15), resección de septo intrauterino asociada con procedimiento de inyección de esperma intracitoplasmática $(16,17)$ y la fertilización in vitro (18).

También se han reportado en la literatura sobre el tema otros procedimientos no asociados con tratamientos de infertilidad, y ejemplo de ello son el curetaje endometrial (19), la cesárea segmentaria (20), la colposcopia con diatermia (21), el curetaje obstétrico posparto por retención de placenta (22) y la histeroscopia con miomectomía (23). Finalmente, se describen algunas otras asociaciones, como las adherencias intrauterinas, el síndrome de Asherman, la utilización de dispositivos intrauterinos (24), la endometritis crónica y la exposición in utero a Dietiletilbestrol.

\section{Aproximación diagnóstica}

\section{Ecografia endovaginal}

El uso de la ecografía endovaginal ha permitido un diagnóstico rápido y temprano del EEC, con la posibilidad de optar por tratamientos conservadores en estas pacientes. El ultrasonido como técnica para el diagnóstico del EEC se reporta desde 1978 (24).

Su implementación como método de diagnóstico en patología ginecológica y obstétrica hoy es universal y no cabe duda de que permitió comprender y ampliar el conocimiento de esta condición rara y potencialmente grave. Los criterios ecográficos para el diagnóstico de EEC fueron propuestos por Timor-Tritsch y cols. (10), en 1994, y Jurkovic y cols. (25), en 1996. Se pueden resumir de la siguiente forma:

1. Presencia de cavidad endometrial vacía.

2. Cuello uterino en forma de barril.

3. Presencia de saco gestacional por debajo del nivel de las arterias uterinas.

4. Ausencia de "signo de deslizamiento", donde a la presión con el transductor vaginal hay movimiento del saco gestacional en los casos de aborto en curso, lo cual lo diferencia con el ectópico cervical, donde no observamos este fenómeno.

5. Presencia de flujo sanguíneo alrededor del saco gestacional en la revisión con una ecografía Doppler color.

El ultrasonido permite hacer la aproximación terapéutica en modelos de manejo que involucran la inyección de medicamentos en el saco gestacional y el seguimiento en el corto y largo plazo de los resultados de la terapia elegida.

\section{Resonancia magnética nuclear}

El propósito de la RMN es aportar información adicional para mujeres que necesiten un diagnóstico preciso, caracterización del tejido y ubicación espacial. Algunas indicaciones de la resonancia en el EE son las complicaciones asociadas, hallazgos ecográficos no concluyentes o complicaciones del $\mathrm{EE}$, como las localizaciones abdominal, intersticial, miometrial o cervical (26).

La localización cervical de un EE por debajo del orificio cervical interno puede confundirse con un aborto espontáneo en curso, con enfermedad trofoblástica, con un pólipo endometrial que protruye por el canal cervical o con una placenta de implantación baja (27).

Jung y cols. (28) encontraron como hallazgos en RMN de EEC una masa mal definida con señales muy heterogéneas en $\mathrm{T} 2$, hiperintensidades internas e irregulares en T1, con un anillo circunferencial o parcial de baja intensidad de señal, con realce periférico irregular del componente sólido papilar, acompañado de vacíos de señal de morfología tubular. Esto va en concordancia con lo referido por Tamai y cols. (27), que describen típicamente el EEC como una masa hemorrágica, heterogénea y con realce intenso de los componentes papilares del tejido.

Una secuencia útil en la identificación del hematoma reciente es el $\mathrm{T} 2^{*}$, que podría remplazar el uso del gadolinio en esta indicación particular. La sensibilidad del 95\% y la especificidad del $100 \%$ de esta secuencia mejora significativamente el desempeño de la resonancia en el diagnóstico de $\mathrm{EE}$ (29). 
El uso de gadolinio intravenoso incrementa la especificidad en el diagnóstico de embarazo tubárico, lo que permite diferenciar el realce normal de la trompa de un hematoma reciente. En este caso, este principio nos ayudó a delimitar la afectación decidual y a descartar la presencia de un hematoma asociado (30). La resonancia con gadolinio también se ha utilizado en el seguimiento del tratamiento conservador del EEC (31).

\section{Tratamiento}

De acuerdo con la descripción de Studdiford (32), de la Universidad de Columbia, en 1945, el EEC era una condición muy rara, sino inexistente, reportada en una sola oportunidad en la literatura escrita del siglo XX. Debido a los problemas para el diagnóstico y la elevada letalidad, la evidente naturaleza de esta condición fue claramente aceptada cuatro años después (33).

El tratamiento quirúrgico era la única posibilidad de tratamiento para estas pacientes; sin embargo, con el uso de la ecografía temprana, la detección inicial de estos casos con presencia de sacos gestacionales menores a 12 semanas, la ausencia de actividad cardiaca y bajas cantidades beta de la gonadotrofina coriónica humana hicieron del tratamiento conservador una posibilidad terapéutica (34).

En efecto, la introducción de la ecografía mejoró el panorama diagnóstico, y la condición fue cada vez más fácilmente identificada. La manipulación quirúrgica como primera opción en el EEC confirmado genera un riesgo importante de complicaciones. Hosni y cols. (35) publicaron una revisión sistemática de la literatura donde analizaron las distintas estrategias diagnósticas y de la cual se pueden derivar algunas conclusiones importantes:

Los reportes de caso y las series de pacientes coinciden en una menor tasa de complicaciones cuando el diagnóstico se hace durante el primer trimestre a cuando este se realiza después de la semana doce de gestación.

Una tendencia a mejorar la efectividad del manejo médico existe en presencia de óbito embrionario u óbito fetal, a pesar de que la información se deriva de la revisión de muchos reportes de casos y algunas series pequeñas. Esto parece ser un factor determinante del fracaso terapéutico en algunas pacientes, la viabilidad embrionaria o fetal.

El propósito del manejo conservador, entendido como una serie de estrategias médicas o quirúrgicas y la combinación de estas, debe ser minimizar el sangrado, eliminar los productos de la gestación y conservar el útero o la capacidad reproductiva.

En cuanto al tipo de manejo, se describe como radical la amputación quirúrgica del cuello uterino (traquelectomía) o la histerectomía abdominal total. $\mathrm{Al}$ contrario, el tratamiento conservador, que busca preservar la función, es una opción terapéutica (médica o quirúrgica) que se puede ofrecer a pacientes que desean conservar su fertilidad.

Los esquemas publicados para manejo médico o quirúrgico conservador del EEC son variados y muchos son el reflejo de esquemas descritos como útiles para el EE tubárico, e incluyen la inyección directa de cloruro de potasio (36), la actinomicina D (37), la mifepristona (38), la combinación de algunos (39) y la inyección de prostaglandinas $(40,41)$.

Los estudios sobre los distintos esquemas medicamentosos son más numerosos por obvias razones en el grupo del EE tubárico, y la estrategia terapéutica elegida para EEC usualmente se deriva de los mencionados. Es claro, sin embargo, que dentro de las opciones terapéuticas de manejo conservador, el metotrexato está en primera línea. Marret (42), en una revisión narrativa sobre su utilización en forma off label destaca que es la primera opción de tratamiento para gestantes con EEC. Su administración puede ser sistémica, y por medio de inyección local, advirtiendo la posibilidad de necesidad de embolización o el riesgo de histerectomía. El artículo sugiere para EE una dosis de $1 \mathrm{mg} / \mathrm{kg}$ y la necesidad de realizar pruebas de función hepática y renal antes del tratamiento.

El metotrexato es un inhibidor de la dihidrofolato reductasa, que produce una interrupción en la síntesis de nucleótidos, como la purina, y de aminoácidos, como serotonina y metionina, al inhibir la síntesis de ADN y la replicación y reparación celular (43).

El metotrexato sistémico se ha usado para el tratamiento de la enfermedad trofoblástica gestacional desde 1956 (44) y un reporte de su 
utilización por primera vez para el tratamiento del EE data de 1982 (45).

El esquema que se utilizó en nuestra paciente fue de múltiples dosis, debido a los niveles de beta de la gonadotrofina coriónica humana en el momento del diagnóstico. El esquema recomendado es de una dosis diaria de metotrexato de $1 \mathrm{mg} / \mathrm{kg}$ de peso los días 1, 3, 5 y 7 , alternándolo con ácido folínico a dosis de $0,1 \mathrm{mg} / \mathrm{kg}$ de peso los días 2, 4, 6 y 8 con control de las concentraciones de beta de la gonadotrofina coriónica humana entre el día 4 y 7 de tratamiento, esperando una disminución de más del $15 \%$ para considerar la eficacia terapéutica $(46,47,48)$.

La tasa de éxito con el tratamiento en múltiples dosis es de aproximadamente el 93\%, comparada con un $81 \%$ en pacientes con una dosis única. Se evidencia una persistencia del trofoblasto en un $8 \%$ contra un $4 \%$ en pacientes con dosis única vs. dosis múltiple, respectivamente $(46,47)$.

Podemos afirmar entonces que el éxito terapéutico depende del régimen usado, de la edad gestacional y de las concentraciones beta de la gonadotrofina coriónica humana $(46,48)$. No hay un protocolo estándar para el uso de metotrexato en pacientes con EEC: algunas pacientes requieren maniobras adicionales, como la administración local de metotrexato o de cloruro de potasio, en presencia de un embrión vivo.

Son predictores de mala respuesta al manejo del EEC una edad gestacional mayor de 9 semanas; las concentraciones beta de la gonadotrofina coriónica humana mayores a $10.000 \mathrm{mUI} / \mathrm{ml}$; una longitud cráneo-caudal mayor de $10 \mathrm{~mm}$, y actividad cardiaca fetal. No hay una clara recomendación sobre la dosis óptima de administración para el tratamiento del $\operatorname{EEC~(34).~}$

\section{Conclusiones}

El diagnóstico temprano del EE tubárico y extratubárico es básico para ofrecerle a la paciente un tratamiento conservador que, ayudado por RMN, permita preservarle su fertilidad con buenos resultados clínicos.

La importancia de un reconocimiento preciso del EEC permite establecer un modelo de manejo conservador que disminuya las complicaciones ocasionalmente catastróficas de esta condición o su inapropiado enfoque terapéutico $(49,50)$.

A pesar de ser una condición rara, el especialista debe estar en condiciones de reconocer adecuadamente y actuar en conformidad frente al riesgo de EEC.

\section{Conflictos de interés}

Ninguno declarado.

\section{Agradecimientos}

El presente artículo fue logrado mediante la utilización de recursos documentales de la Pontificia Universidad Javeriana, de recursos propios de los autores y no contó con ninguna fuente externa de financiación.

\section{Referencias}

1. 1. National Center for Biotechnology Information. Pregnancy, ectopic [internet]. Disponible en: http://www.ncbi.nlm.nih.gov/mesh /?term $=$ ectopic + pregnancy.

2. 2. Confidential Enquiry into Maternal and Child Health. Saving mothers' lives: Reviewing manternals deaths to make motherhood safer: 2003-2005 [internet]. London: CEMACH; 2007. Disponible en: http://www.publichealth.hscni.net/ sites/default/files/Saving\%20Mothers \%27\%20Lives\%202003-05\%20.pdf

3. 3. Centers for Disease Control and Prevention (CDC). Ectopic pregnancy -United States, 1990-1992. MMWR Morb Mortal Wkly Rep. 1995 Jan 27;44(3):46-8.

4. 4. Goldner TE, Lawson HW, Xia Z, Atrash HK. Surveillance for ectopic pregnancy--United States, 1970-1989. MMWR CDC Surveill Summ. 1993 Dec 17;42(6):73-85. 
5. 5. Ruiz A. Embarazo ectópico. En: Ángel E, editor. Obstetricia integral: siglo 21. Bogotá: Universidad Nacional de Colombia; 2009.

6. 6. Rodríguez DA, Acosta J. Panorama de la mortalidad materna en Colombia, 1995-2000: logros, fracasos, compromisos y retos. Inf Quinc Epidemiol Nac. 2001;6(23)-345-60.

7. 7. Kirk E, Condous G, Haider Z, Syed A, Ojha K, Bourne T. The conservative management of cervical ectopic pregnancies. Ultrasound Obstet Gynecol. 2006;27:430-7.

8. 8. Oliver R, Malik M, Coker A, Morris J. Management of extra-tubal and rare ectopic pregnancies: case series and review of current literature. Arch Gynecol Obstet. 2007;276:125-31.

9. 9. Gómez JA. 95 casos de embarazo ectópico. Rev Colomb Obstetr Ginecol. 1954;4:17-89.

10. 10. Timor-Tritsch IE, Monteagudo A, Mandeville EO, Peisner DB, Anaya GP, Pirrone EC. Successful management of viable cervical pregnancy by local injection of methotrexate guided by transvaginal ultrasonography. Am J Obstet Gynecol. 1994 Mar;170(3):737-9.

11. 11. Herrera E, Otero E, Hincapié LC, Camacho R, Gómez G, Quintero $\mathrm{CH}$, et al. Heterotopic pregnancy: Presentation of four cases. Colomb Med [internet].\# 2011\#[citado\# 2015 \# Feb\# 22];42(4):518-22. Disponible en: http://www.scielo.org.co/scielo.php ?script $=$ sci_arttext\&pid $=$ S1657-9534 $2011000400013 \& \operatorname{lng}=$ en .

12. 12. Verma U, Goharkhay N. Conservative management of cervical ectopic pregnancy. Fertil Steril. 2009 Mar;91(3):671-4. doi: 10,1016/ j.fertnstert.2007,12,054.

13. 13. Brinsden PR, Smith B, Craft IL. Cervical ectopic pregnancy following gamete intrafallopian transfer. J In Vitro Fert Embryo Transf. 1988 Dec;5(6):370-2.

14. 14. Balasch J, Peñarrubia J, Ballescá JL, Creus M, Casamitjana R, Vanrell JA. Intra-uterine insemination, cervical pregnancy and successful treatment with methotrexate. Hum Reprod. 1994 Aug;9(8):1580-3.

15. 15. Giarenis I, Shenoy J, Morris E. Cervical ectopic pregnancy after endometrial ablation: A case report. Arch Gynecol Obstet. 2008 Jun;277(6):567-9.

16. 16. Ahmadi F, Irani S. Cervical ectopic pregnancy following assisted reproductive technology: A case report. Int J Fertil Steril. 2012 Oct;6(3):201-4.

17. 17. Pantelis A, Daniilidis A, Dinas K. Conservative treatment of a 7 weeks cervical ectopic pregnancy after intrauterine insemination. Hippokratia. 2013 Jan;17(1):95.

18. 18. Anev I, Wang J, Palep-Singh M, Seif MW. Monochorionic diamniotic twin cervicalectopic pregnancy following assisted conception: a case report. J Reprod Med. 2013 SepOct;58(9-10):445-7.

19. 19. Giorgio B, Ball KG, Ogden S, Verco PW, Finikiotis G. Cervical ectopic pregnancy--case report. Aust N Z J Obstet Gynaecol. 1980 Aug;20(3):164-7.

20. 20. Hingorani SR, Parulekar SV, Ratnam KL. Isthmico-cervical ectopic pregnancy following caesarean section. J Postgrad Med. 1994 JanMar;40(1):33-5.

21. 21. Gun M, Mavrogiorgis M. Cervical ectopic pregnancy: a case report and literature review. Ultrasound Obstet Gynecol. 2002 Mar;19(3):297-301.

22. 22. Cerveira I, Costa C, Santos F, Santos L, Cabral 
F. Cervical ectopic pregnancy successfully treated with local methotrexate injection. Fertil Steril. 2008 Nov;90(5):2005.e7-2005.e10. doi: 10.1016/j.fertnstert.2008.01.017.

23. 23. Yu B, Douglas NC, Guarnaccia MM, Sauer MV. Uterine artery embolization as an adjunctive measure to decrease blood loss prior to evacuating a cervical pregnancy. Arch Gynecol Obstet. 2009 May;279(5):721-4. doi: 10.1007/ s00404-008-0775-4.

24. 24. Raskin MM. Diagnosis of cervical pregnancy by ultrasound: a case report. Am J Obstet Gynecol. 1978 Jan 15;130(2):234-5.

25. 25. Jurkovic D, Hacket E, Campbell S. Diagnosis and treatment of early cervical pregnancy: a review and a report of two cases treated conservatively. Ultrasound Obstet Gynecol. 1996 Dec;8(6):373-80.

26. 26. Nagayama M, Watanabe Y, Okumura A, Amoh Y, Nakashita S, Dodo Y. Fast MR imaging in obstetrics. Radiographics. 2002 MayJun;22(3):563-80.

27. 27. Tamai K, Koyama T, Togashi K. MR features of ectopic pregnancy. Eur Radiol. 2007 Dec;17(12):3236-46.

28. 28. Jung SE, Byun JY, Lee JM, Choi BG, Hahn ST. Characteristic MR findings of cervical pregnancy. J Magn Reson Imaging. 2001;13(6):918-22.

29. 29. Yoshigi J, Yashiro N, Kinoshita $T$, O'uchi T, Kitagaki H. Diagnosis of ectopic pregnancy with MRI: efficacy of T2*-weighted imaging. Magn Reson Med Sci. 2006 Apr;5(1):25-32.

30. 30. Kataoka ML, Togashi K, Kobayashi $\mathrm{H}$, Inoue $\mathrm{T}$, Fujii $\mathrm{S}$, Konishi $\mathrm{J}$. Evaluation of ectopic pregnancy by magnetic resonance imaging. Hum Reprod. 1999 Oct;14(10):2644-50.
31. 31. Takashima M, Yamasaki M, Fujita I, Ohashi M, Matsuo H, Mochizuki M, Kitagaki H, Kono M. Enhanced magnetic resonance imaging in monitoring of conservative treatment of cervical pregnancy. J Obstet Gynaecol. 1995 Dec;21(6):545-50.

32. 32. Studdiford WE. Cervical pregnancy: A partial review of the literature and a report of two probable cases. Am J Obstet Gynecol. 1945;49(2):169-85.

33. 33. Morton DG. Cervical pregnancy; a report of two cases and a discussion of the treatment. Am J Obstet Gynecol. 1949 May;57(5):910-7.

34. 34. Chetty M, Elson J. Treating non-tubal ectopic pregnancy. Best Pract Res Clin Obstet Gynaecol. 2009;23(4):529-38. doi: 10.1016/ j.bpobgyn.2008.12.011

35. 35. Hosni MM, Herath RP, Mumtaz R. Diagnostic and therapeutic dilemmas of cervical ectopic pregnancy. Obstet Gynecol Surv. 2014 May;69(5):261-76. doi: 10.1097/ OGX.0000000000000062.

36. 36. Aboulghar MA,Mansour RT,\#Serour GI. Transvaginal injection of potassium chloride and methotrexate for the treatment of tubal pregnancy with a live fetus.\#Hum Reprod. 1990;5:887-8.

37. 37. Altaras M,Cohen I,Cordoba M,Ben-Nun I, Ben-Aderet N. Treatment of an interstitial pregnancy with actinomycin $\mathrm{D}$ : case report.Br J Obstet Gynaecol.\#1988;95:1321-3.

38. 38. Gazvani MR,\#Emery SJ. Mifepristone and methotrexate: the combination for medical treatment of ectopic pregnancy.Am J Obstet Gynecol.\#1999;180:1599-1600.

39. 39. Kenigsberg D,\#Porte J,Hull M,\#Spitz IM. Medical treatment of residual ectopic pregnancy: RU 
486 and methotrexate.\#Fertil Steril. 1987;47:702-3.

40. 40. Lindblom B,\#Hahlin M,\#Lundorff P,\#Thorburn J. Treatment of tubal pregnancy by laparoscope-guided injection of prostaglandin F2 alpha.\#Fertil Steril.\#1990;54:404-8.

41. 41. Lang PF,\#Weiss PA,\#Mayer $\mathrm{HO}$,\#Haas JG, Honigl W. Conservative treatment of ectopic pregnancy with local injection of hyperosmolar glucose solution or prostaglandinF2 alpha: A prospective randomised study.\#Lancet.\#1990;336:78-81.

42. 42. Marret $\mathrm{H}$, Fauconnier $\mathrm{A}$, Dubernard G, Misme H, Lagarce L, Lesavre M, et al.. Overview and guidelines of off-label use of methotrexate in ectopic pregnancy: Report by CNGOF. Eur J Obstet Gynecol Reprod Biol. 2016;205:105-9.

43. 43. Stika CS. Methotrexate: the pharmacology behind medical treatment for ectopic pregnancy. Clin Obstet Gynecol. 2012 Jun;55 (2):433-9. doi: 10.1097/GRF.0b013e3182510a35.

44. 44. Hertz R, Li MC, Spencer DB. Effect of methotrexate therapy upon choriocarcinoma and chorioadenoma. Proc Soc Exp Biol Med. 1956 Nov;93(2):361-6.

45. 45. Tanaka T, Hayashi H, Kutsuzawa T, Fujimoto S, Ichinoe K. Treatment of interstitial ectopic pregnancy with methotrexate: report of a successful case. Fertil Steril. 1982 Jun;37(6):851-2.

46. 46. American College of Obstetricians and Gynecologists. ACOG Practice Bulletin No. 94: Medical management of ectopic pregnancy. Obstet Gynecol. 2008 Jun;111(6):1479-85.

47. 47. Varma R, Mascarenhas L. Evidence-based management of ectopic pregnancy. Reprod Med Rev. 2001;9(1):49-75.
48. 48. Mitra AG, Harris-Owens M. Conservative medical management of advanced cervical ectopic pregnancies. Obstet Gynecol Surv. 2000 Jun; 55(6):385-9.

49. 49. Chrestiana D, Cheng AB, Panebianco NL, Dean AJ. Pitfalls in cervical ectopic pregnancy diagnosis by emergency physicians using bedside ultrasonography. Am J Emerg Med. 2014 Apr;32(4):397.e1-3. doi: 10.1016/j.ajem.2013.10.055.

50. 50. Vela G, Tulandi T. Cervical pregnancy: the importance of early diagnosis and treatment. J Minim Invasive Gynecol. 2007 JulAug;14(4):481-4. 